Education

\section{Are paediatricians failing at school?}

\section{J M Goepel, K D Forsyth}

\section{Paediatricians need to understand the morbidity of children with special educational needs}

$\mathrm{T}$ he notion that paediatricians act in the interests of the child to facilitate optimal outcomes is well entrenched within the medical culture. From this perspective paediatricians act to provide curative interventions in reversible situations, and to provide assistance and support for those children with chronic conditions where a return to full functionality is improbable.

There has however been a shift in the spectrum of child health problems seen by paediatricians in recent years. The previous sharp demarcation between readily reversible acute child health problems and the chronic conditions and disabilities of childhood have been supplemented by increasing numbers of children with special educational needs (SEN). Section 312 of the 1996 UK Education Act states that:

"Children have special educational needs if they have a learning difficulty which calls for special educational provision to be made for them"

Children with SEN are just as functionally compromised and in need of paediatrician support as those with organic pathology. Has paediatrics and the practice of paediatricians sufficiently adjusted to these changes? Are paediatricians sufficiently cognisant of the requirements of children with SEN to provide the supportive interventions that they so ably provide for those with organic pathology?

Children with SEN are frequently first identified in early education settings or primary schools. The 1997 DfEE Green paper ${ }^{1}$ points out that "schools currently identify $18 \%$ of children as having special educational needs of some kind". School teachers are in an excellent position to identify such needs in children, as they see the child for prolonged periods each day. Experienced teachers have a finely tuned perspective on the range of normality. They meet the parents or carers, so understanding the social context of the child. The UK government has given high priority to ensure that children with SEN are enabled with appropriate service provision:

"We want to focus on identifying the needs of children with SEN and placing them at the centre of service provision, rather than concentrating on the different statutory responsibilities of individual agencies ${ }^{\prime 2}$

Schools are held accountable for the education of children through legislation, yet struggle to meet the educational requirements for children with SEN.

Only a few of the $18 \%$ of children with SEN have a medical component to this need, thereby requiring to be seen by a paediatrician. Approximately $2 \%$ are statemented. Schools seek assistance for medical assessments and reports in the statementing process, for diagnosis of certain medical conditions, and for ongoing advice and support for the very small number of children with long term medical needs.

Do paediatricians have any statutory responsibilities for such children? The 1989 Children Act (section 27) requires

"Local Education Authorities and District Health Authorities to provide services which are considered necessary for children identified as being 'in need"'

Hence both local education authorities and district health authorities have responsibility for assisting such children. Paediatricians see children in a consultative environment and in general ensure other agencies are providing the necessary supports, but are often not cognisant of educational legislative requirements for children. Section 322 of the 1996 Education Act states:

"Health Authorities, subject to the the light of the resources available to them, must comply with a request for help from an LEA in connection with children with special educational needs, unless they consider that the help is not necessary for the exercise of the LEA's functions"

Furthermore, the UK government's SEN Code of Practice ${ }^{3}$ provides practical guidance concerning Part IV of this Act. This advice is for LEAs, all providers of education "and those who help them, including health and social services". All of reasonableness of the request in these agencies "must have regard to" the Code. "They must not ignore it". Therefore it should be understood that there is an undeniable requirement on paediatricians to be assistive to the school in their endeavours to meet the needs of the child.

The mandate of the school is to optimise learning outcomes for the child, and, as in paediatrics, they seek to develop the child to their fullest potential. To achieve this, schools need good collaboration with the health services, particularly paediatricians. Schoolteachers, whose task is to implement education for children with SEN, rely heavily on the health services for assessments, diagnosis, ongoing support to families, and appropriate intervention strategies. Underpinning this engagement between health and education is the understanding that "health and wellbeing is inseparable from learning and developing for a future". ${ }^{4}$ The SEN Code of Practice states:

"All agencies should recognise the need for effective collaboration of services involved with the child and the parents. Consultative responsibilities and effective communication systems at management and practitioner levels should be clearly identified. Developments and organisational structures and working practices should reflect this principle " $^{3}$

Hence for optimal outcomes for children with SEN it is imperative that the health service works closely with education. However, such a partnership between health and education is far from straightforward. Professor Philip Graham, Chair of the National Children's Bureau in London states:

"There are similarities and differences between the two worlds of education and health. In both worlds the professionals want the best for children, but sometimes cooperation between them is made difficult by barriers, including different concepts and languages, financial pressures, lack of time to communicate and the lack of shared evidence base ${ }^{\prime \prime 5}$

Recent research into the effectiveness of collaboration between health and education showed major deficiencies:

"Good communication and effective collaboration between schools and health, social and other agencies are not prevalent. When they do occur they depend largely on local personal contacts, they are fraught with communication difficulties and concerns over funding ${ }^{\prime \prime 6}$ 
Paediatricians may then by default be failing to assist children in optimising their educational opportunities at school. As paediatricians become increasingly involved in this new morbidity of childhood, what is needed is greater understanding of the different cultures between these two agencies and a greater understanding by the health system of the requirements of schools. Above all it is imperative that the child becomes central, so that the agencies involved in provision for that child can cooperate in a manner which optimises the outcomes for the child rather than for the service. How can this be achieved and what practical steps can paediatricians and schools take to enable more effective partnership? Paediatricians need to understand their pivotal role in providing information and strategies to support the school and their professional counterparts in education, as well as the child. Such understanding would lead to timely responses by paediatricians to the school in response to requests for information and advice regarding pupils. There would be a willingness by paediatricians to listen to and give credence to concerns from school regarding children, and the provision of clear, specific, and relevant reports to aid reviews and the statementing process. Only by these two key professional groups sharing assessments, perspectives, and opinion, can a consensus of need be reached, thereby facilitating a unified understanding, a common approach, and effective strategies.

Another professional group who have an important role for children with SEN are the educational psychologists. They carry out assessments and offer interventions for some children. They are able to share their findings with paediatricians, avoiding duplication. Their role is complementary to paediatricans, but they are not medically qualified and so are unable to provide the comprehensiveness of a medical assessment. There is however a shortage of educational psychologists, exacerbating the problems of assessment of children with SEN.

Training of paediatricians should ideally include exposure to children with SEN in the school environment, and some rudimentary understanding of the legislative environment of education, which will enable more appropriate reports and collaboration between the two services. As children with SEN touch every aspect of paediatrics, such training should not be restricted to community paediatrics.

How can collaboration be enhanced? In the DfEE Publication Effective communication between schools, LEAs and health and social services in the field of special educational needs ${ }^{7}$ some suggestions are provided, including:

- The effective management and transfer of information between agencies. This should include, where appropriate, signed informed consent by parents for the transfer of information on their child between health and education.

- Each agency to have a publicly available statement on the value they place on interagency cooperation, with annually reviewable targets.

- Training and staff development (possibly jointly, to promote greater understanding).

- Planning and monitoring of progress. Joint targets and goals for children with SEN to be subject to interagency monitoring.

"Effective inter-agency co-operation therefore depends crucially on the development of a 'multi-dimensional gaze' through which individual professionals and agencies as a whole can see problems from each other's perspectives, appreciate and utilise each other's expertise, and come to some shared understanding of children's needs ${ }^{\prime \prime}$

Paediatricians need to ensure they understand the morbidity of children with SEN, that they support the schools' efforts in provision of education, and that they are not failing the school or, more importantly, the child.

Arch Dis Child 2002;87:173-174

Authors' affiliations

J M Goepel, SEN Co-ordinator, Lydgate Junior School, Sheffield, UK

K D Forsyth, Professor of Paediatrics, Sheffield Children's Hospital, Sheffield, UK, and Flinders University, South Australia

Correspondence to: Professor K D Forsyth, Department of Paediatrics, Flinders Medical Centre, Adelaide 5042, South Australia, Australia; kevin.forsyth@flinders.edu.au

\section{REFERENCES}

1 DfEE. Excellence for all children: meeting special educational needs. London: $\mathrm{HMSO}$ 1997:4.

2 DfEE. Meeting special educational needs: a programme of action. London: HMSO, 1998:33.

3 DfES. Special Educational Needs Code of Practice. Nottingham: DfES Publications, 2001.

4 Anderson $M$. What does the health and education interface look like? Health Education Australia 2001:1:30-2.

5 Graham P. Royal Children's Hospital Education Institute Annual Report. Melbourne, Victoria, Australia, 2000:2

6 Daniels H, Visser J, Cole T, et al. Emotional and behavioural difficulties in mainstream schools. In Research Brief No. 90. 1999. http://www.dfee.gov.uk/research/re_brief/ RB60.doc

7 Dyson A, Lin M, Milward A. Effective communication between schools, LEAs and the Health and Social Services in the field of special needs. Sudbury: DfEE Publications, 1998. 